\title{
Endogenous $\mathrm{n}-3$ polyunsaturated fatty acids
} (PUFAs) mitigate ovariectomy-induced bone loss by attenuating bone marrow adipogenesis
in FATI transgenic mice

This article was published in the following Dove Press journal:

Drug Design, Development and Therapy

27 June 2013

Number of times this article has been viewed

\author{
Tian-yu Chen ${ }^{1,2, *}$ \\ Zhong-min Zhang ${ }^{1,2, *}$ \\ Xiao-chen Zheng ${ }^{1,2}$ \\ Liang Wang ${ }^{1,2}$ \\ Min-jun Huang ${ }^{1,2}$ \\ Si Qin ${ }^{3}$ \\ Jian Chen ${ }^{1,2}$ \\ Ping-lin Lai ${ }^{4}$ \\ Cheng-liang Yang ${ }^{1,2}$ \\ Jia Liu ${ }^{1,2}$ \\ Yi-fan Dai ${ }^{5}$ \\ Da-di Jin ${ }^{1,2}$ \\ Xiao-chun Bai ${ }^{1,2,4}$
}

'Department of Orthopaedic, the Third Affiliated Hospital of Southern Medical University, Guangzhou, Guangdong,

People's Republic of China; ${ }^{2}$ Academy of Orthopaedics, Guangdong Province,

Guangzhou, Guangdong, People's Republic of China; ${ }^{3}$ Department of Dermatology and STD, Guangdong No.2 Provincial People's Hospital, Guangzhou, Guangdong, People's Republic of China; ${ }^{4}$ Department of Cell Biology, School of Basic Medical Science, Southern Medical University, Guangzhou, Guangdong, People's Republic of China; ${ }^{5}$ Center of Metabolic Disease Research, Nanjing Medical University, Jiangsu, People's Republic of China

*These authors contributed equally to this work

Correspondence: Xiao-chun Bai/Da-di Jin Department of Cell Biology, School of Basic Medical Science, Southern Medical University, 1838 North Guangzhou Avenue, Guangzhou 510515,

People's Republic of China

Tel +862061648724

Fax +862061648208

Email xiaochun_bai@I63.com/baixcl5@ smu.edu.cn
Aim: To investigate the effect of endogenous n-3 polyunsaturated fatty acids (PUFAs) on bone marrow adipogenesis under osteoporosis conditions.

Methods: A mouse osteoporosis model overexpressing the FAT1 gene from Caenorhabditis elegans and converting n-6 PUFAs to n-3 PUFAs endogenously was used.

Results: The mice presented significantly lower bone marrow adiposity (adipocyte volume/tissue volume, mean adipocyte number) but increased the bone parameters (bone mineral density, bone mineral content, bone volume/total volume) in the distal femoral metaphysis.

Conclusion: Endogenous n-3 PUFAs protect bone marrow adipogenesis, which provides a novel drug target.

Keywords: antiosteoporosis, n-3 PUFAs, bone marrow, adipogenesis

\section{Introduction}

Osteoporosis is a common clinical disease in postmenopausal women and aging populations, is caused by an imbalance between bone resorption and bone formation, and leads to osteoporotic fractures and other serious complications. ${ }^{1}$ Age-induced osteoporosis is mainly associated with increased bone marrow adipocytes and bone loss. ${ }^{2,3}$ In osteoporotic bone, reduced bone formation is directly correlated with increasing adipose tissue. $^{4}$

Osteoblasts and marrow adipocytes are both differentiated from a common precursor: bone marrow mesenchymal stem cells (BMSCs). ${ }^{5}$ An in vitro study has revealed that mesenchymal stem cells (MSCs) derived from postmenopausal women with osteoporosis are more likely to differentiate into adipocytes than osteoblasts, compared with cells isolated from healthy women. ${ }^{6}$ There is virtual redifferentiation plasticity in both osteoblasts and adipocytes. It has been demonstrated that fully differentiated osteoblasts from human MSCs presented capabilities of differentiation and transdifferentiation into adipocytes and vice versa. ${ }^{7}$ It also has been reported that the differentiation of MSCs into adipocytes or osteoblasts is competitively balanced within the bone marrow. ${ }^{8}$ Beresford and coworkers have shown the inverse relationship between the differentiation of adipocytic and osteogenic cells. ${ }^{9}$ Moreover, Clabaut et al proposed that MSC-derived adipocytes are able to induce MSC-derived osteoblasts to differentiate to an adipocyte phenotype within an in vitro coculture system. ${ }^{10} \mathrm{On}$ the other hand, various studies have indicated that adipocytes can secrete factors, such as hormones, leptin, adiponectin, and fatty acids, which affect the prolifera- 
tion, apoptosis, and function of neighboring cells. ${ }^{11,12}$ The evidence from the investigation conducted by Elbaz et al suggests an effect of lipotoxicity from marrow adipocytes on osteoblast function. ${ }^{13}$ In addition, cross talk between adipocytes and osteoclasts has been unearthed recently. It has been reported that preadipocytes have a stronger ability in supporting osteoclast-like cell formation without prostaglandin E2 than preosteoblasts, suggesting an emerging relationship between bone marrow-cell adipogenesis and osteoclastogenesis. ${ }^{14}$

The existing evidence delineates that bone marrow-fat accumulation may play a critical role in the onset of osteoporosis, owing to its potential effects on either the commitment of BMSC differentiation or the homeostasis of osteoclastmediated bone resorption. Based on this notion, reversal bone marrow adipogenesis may be an effective therapeutic strategy for the prevention of age-related osteoporosis.

N-3 long-chain polyunsaturated fatty acids (PUFAs) are essential fatty acids for human beings, and include alphalinolenic acid, eicosapentaenoic acid, and docosahexaenoic acid, which cannot be self-synthesized in mammals. ${ }^{15}$ During the past few decades, n-3 PUFAs have been supposed to be beneficial for prevention from bone loss in both in vitro and in vivo studies. However, the underlying mechanisms of this action have not yet been fully defined. ${ }^{16-22}$ A recent study has identified that inhibition of osteoclast differentiation and activation may be one of the mechanisms involved in preventing osteoporosis by n-3 PUFAs $(\mathrm{n}-6: \mathrm{n}-3=1: 1){ }^{23}$

To the best of our knowledge, there are no reports regarding the roles and mechanisms of n-3 PUFAs on bone marrow adipogenesis. In the present study, by employing a transgenic (TG) mouse model that overexpresses the FAT1 gene from Caenorhabditis elegans and converts n-6 PUFAs to n-3 PUFAs endogenously at a fixed ratio compared to other diet-formulated approaches, we aimed firstly to investigate the effect of endogenous n-3 PUFAs on bone marrow adipogenesis under osteoporosis conditions, which could give rise to a novel insight into current therapy for antiosteoporosis. Peroxisome proliferator-activated receptor gamma (PPAR $\gamma$ ) is an essential transcription factor in adipogenesis, ${ }^{24}$ the expression and/or activity of which determines the commitment of BMSCs to osteoblasts or adipocyte lineages. ${ }^{25}$ Runt-related transcription factor 2 (RUNX2) is a key regulator of osteoblast differentiation, playing an important role in bone formation, ${ }^{26}$ which was used as the measure of adipogenesis/osteogenesis in the present study.

\section{Materials and methods}

\section{Animal model and diet feeding}

FAT1 TG mice and C57BL/6 wild-type (WT) control mice were donated by Professor Yifan Dai. Since the FAT1 gene is a kind of heterozygote gene, breeder mice were mated with WT C57BL/6 mice to obtain female FAT1 gene-positive C57BL6 mice and FAT1 gene-negative C57BL/6 mice screened by genotyping using a polymerase chain reaction kit (Takara, Dalian, People's Republic of China) (Figure 1). The amount of n-3 PUFAs and n-6 PUFAs in mouse tails were measured by gas-liquid chromatography (Figure 1). The mice were maintained in cages (two to four mice per cage) under controlled laboratory conditions: 12-hour light/ dark cycle at $24^{\circ} \mathrm{C}$, fed normal diets and tap water. At age 2 months, 40 mice (20 FAT1 mice, 20 WT mice) with matched weight received sham or ovariectomy surgeries. Then they were divided into four groups (ten mice/group): group A, FAT1 mice sham; group B, FAT1 ovariectomized (OVX); group C, WT sham; group D, OVX. All animals were fed with standard high-fat diets (Research Diets, New Brunswick, NJ, USA) containing $44.9 \mathrm{kcal} \%$ fat, $35.1 \mathrm{kcal} \%$ carbohydrate, and $20 \mathrm{kcal} \%$ protein. When the mice were 5 months old, they were killed. The final body weight was measured and, bilateral femurs were excised. Then we stripped the soft tissue on bones and immersed them in 4\% paraformaldehyde at $4^{\circ} \mathrm{C}$ for 24 hours.

This study was approved by the Animal Ethics Committee of Southern Medical University, and all experiment procedures strictly followed principles of the National Institutes of Health Guide.

\section{Micro-CT analysis}

Micro-computed tomography (CT) was performed on each bone and focused on distal femoral metaphysis using a ZKKS-MCT-Sharp-III scanner system (Caskaisheng,



Figure I (A and B) Genotyping. (A) PCR genotyping confirmation using FATI fragment-specific primers indicated FATI gene expression (lane I) and wild-type (WT) gene expression (lane 2); lane 3 is the negative control. (B) N-6/n-3 ratio of FATI mice $($ mean $=7.762)$ was significantly lower than WT mice $($ mean $=28.43)$. Note: $* P<0.05$ versus FATI. 
Guangzhou, People's Republic of China). A small field was selected for scanning and was corrected for the $\mathrm{CT}$ value, a 70 $\mathrm{kV}$ scanning voltage, $30 \mathrm{~W}$ power, $429 \mu \mathrm{A}$ current, and $5 \mu \mathrm{m}$ scan thickness. The 3D-MED 3.0 (Institute of Automation, Chinese Academy of Sciences, Beijing, People's Republic of China) was used for 3-D knee reconstruction and image capture. Our analysis included various bone parameters: bone morphometry, bone mineral density (BMD; volumetric), total volume (TV), bone volume (BV), BV/TV, and trabecular number (Tb.N).

\section{Bone marrow adiposity analysis}

The samples of femur bone were processed for decalcification, as previously described, ${ }^{28}$ and then routinely embedded in paraffin. Serial sections $(4 \mu \mathrm{m})$ were obtained from the sagittal plane of the femur condyle. Hematoxylin and eosin and toluidine blue staining were conducted to quantify proximal metaphyseal adipocyte parameters: adipocyte number $\left(\mathrm{AD}^{\#}\right.$, per $\left.\mathrm{mm}^{2}\right)$, percent adipocyte volume per tissue volume (AV/TV). A uniform number of fields were screened in all sections by three individuals, starting three fields from the left end and three fields from the top endocortical surface, ${ }^{29}$ excluding the adipocytes with disruption in the fields. To avoid any bias in the final analysis, all sections were read in a blinded way without knowledge of the groups (FAT1 or WT). Pictures were obtained at $20 \times$ magnifications using an Olympus (Tokyo, Japan) BX51RF stereomicroscope. All measurements were done at 20× magnification using the Image-Pro Plus analysis software (Media Cybernetics, Rockville, MD, USA).

\section{Immunofluorescence and immunohistochemistry analysis for PPAR $\gamma$ and RUNX2 protein expression}

The following primary antibodies were used: rabbit antimouse PPAR $\gamma$ monoclonal antibody (Cell Signaling Technology, Beverly, MA, USA) and rabbit antimouse RUNX2 polyclonal antibody (Bioworld Technology, Louis Park, MN, USA). Tissue sections were incubated in sodium citrate buffer $(0.01 \mathrm{M}, \mathrm{pH}$ 6.0) for 10 minutes to retrieve antigen after deparaffinization and rehydration. The next step was quenching the endogenous peroxidase with 3\% hydrogen peroxide prepared in $100 \%$ ethanol for 5 minutes. Nonspecific binding was blocked by addition of goat serum for 1 hour at room temperature. Then, sections were incubated with primary antibodies - either anti-PPAR $\gamma$ or anti-RUNX 2 - overnight at $4^{\circ} \mathrm{C}$. After being washed in phosphate-buffered saline three times, sections were further incubated with biotinylated secondary antibody (antirabbit immunoglobulin $\mathrm{G}$ ) for 30 minutes at room temperature. Sections were counterstained with hematoxylin. As negative control, nonimmune mouse immunoglobulin $\mathrm{G}$ was used as the primary antibody, and this gave uniformly negative results (data not shown). Pictures were obtained at 20× magnification using an Olympus BX51RF stereomicroscope. The quantification of the level of these two proteins was analyzed by Image-Pro Plus analysis software at $20 \times$ magnification.

\section{Statistical analysis}

Results were expressed as mean \pm standard error of mean, and data were analyzed with the SPSS 13.0 software (IBM, Armonk, NY, USA) using one-way analysis of variance and least significant difference. Post hoc Tukey's tests were performed with SPSS software directly. $P$-values $<0.05$ were considered statistically significant.

\section{Results \\ The effect of endogenous n-3 PUFAs on body weight and abdominal adipose tissue in OVX-induced osteoporosis model}

There was a difference in the weight of uteruses between the OVX and sham groups, which identified the sound establishment of the OVX-induced osteoporosis model in mice. At the beginning of this study, all mice were well matched for age and weight. The values for body weight and abdominal adipose tissue between FAT1 and WT OVX mice were different $(P<0.05) 3$ months postoperatively, but there was no significant difference in body weight between FAT1 and WT sham mice $(P>0.05)$. OVX groups showed higher levels of body weight compared with sham groups (Figure 2).

\section{Effect of endogenous n-3 PUFAs on femur bone microstructure morphometries}

$\mathrm{BMD}, \mathrm{BMC}, \mathrm{BV} / \mathrm{TV}$, and Tb.N in the OVX groups were lower in the femoral region than the sham group. BMD, BV/ $\mathrm{TV}$, and BMC in the distal femur of FAT1 OVX mice were higher $(P<0.05)$ compared to WT OVX mice. However, the Tb.N in the WT OVX group mildly decreased $(P>0.05)$ compared with the FAT1 OVX group. There was no statistical significance between WT and FAT1 sham mice in femur bone microstructure morphometries (Figure 3). 
A

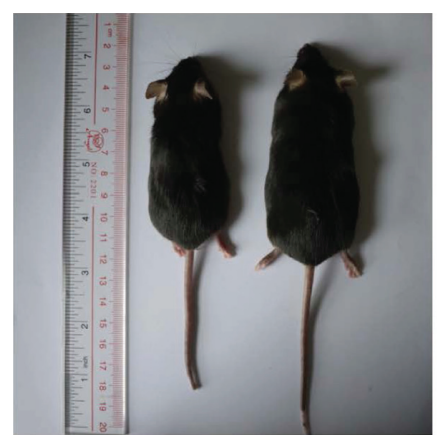

D

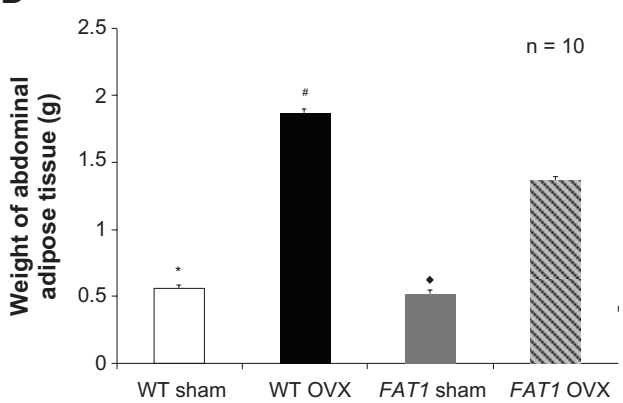

\section{C}
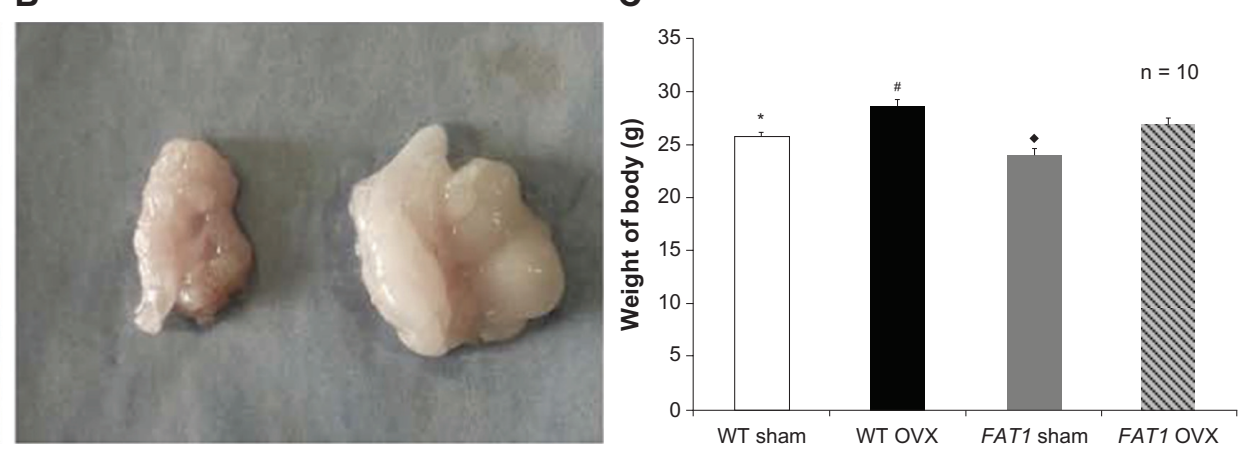

E



Figure 2 (A-E) Gross examination and tissue weight. Three months after ovariectomy, there was increased body weight (C) and abdominal adipose tissue (D) in the ovariectomized (OVX) group compared to the sham group. However, the FATI OVX group presented significant lower body weight (A and C) and abdominal adipose tissue (B and D) compared to the wild-type (WT) OVX group. The weight of uterus was significantly lower in the OVX group than the sham group (E).

Notes: ${ }^{*} P<0.05$ versus WT OVX; ${ }^{*} P<0.05$ versus FATI OVX; ${ }^{*} P<0.05$ versus FATI OVX.

\section{Effect of endogenous n-3 PUFAs on bone marrow adiposity}

The adipocyte parameters in the WT and FAT1 groups are described in Figure 4. There was increased $\mathrm{AD}^{\#}$ in the OVX group compared with the sham group $(P<0.05)$ for both WT and FAT1 mice. Adipocyte parameters such as AV/TV and $\mathrm{AD}^{\#}$ from histological analyses confirmed extensive fat accumulation in the WT OVX group versus the FAT1 OVX group $(P<0.05)$, but the adipocyte parameters between the FAT1 and WT sham groups remained stable (Figure 4).

\section{Effect of endogenous n-3 PUFAs on PPAR $\gamma$ and RUNX2 expression in bone marrow}

In agreement with immunofluorescence data, the intensity of positive staining for PPAR $\gamma$ was enhanced by OVX significantly $(P<0.05)$, and the level of PPAR $\gamma$ protein expression was lower in the FATI OVX group compared with the WT OVX group $(P<0.05)$. There was no significant difference between WT and FAT1 sham mice in the intensity of PPAR $\gamma$-positive staining.
In immunohistochemistry data, positive immunoreaction of RUNX2 appearing in osteoblasts was stronger in the sham group than the OVX group $(P<0.05)$. In the WT OVX group, RUNX2-positive staining was elevated compared with FAT1 OVX mice $(P<0.05)$. There was no statistical significance between WT and FAT1 sham mice in the level of RUNX2 protein expression (Figure 5).

\section{Discussion}

In the present study, the effect of endogenous n-3 PUFAs on bone marrow adipogenesis was investigated in a FAT1 TG OVX model. Our data suggested that TG mice present significantly lower bone marrow adiposity $\left(\mathrm{AV} / \mathrm{TV}, \mathrm{AD}^{\#}\right)$ but increased bone parameters (BMD, BMC, BV/TV) in the distal femoral metaphysis.

Despite the fact that the intimate relationship between bone marrow adipogenesis and bone loss has been realized in osteoporosis pathogenesis, ${ }^{30,31}$ involving several studies on the potential effect of dietary or endogenous n-3 PUFAs on osteogenesis and osteoclastogenesis, ${ }^{32-34}$ the influence of endogenous n-3 PUFAs on adipogenesis in bone marrow is still poorly understood. 
A



B

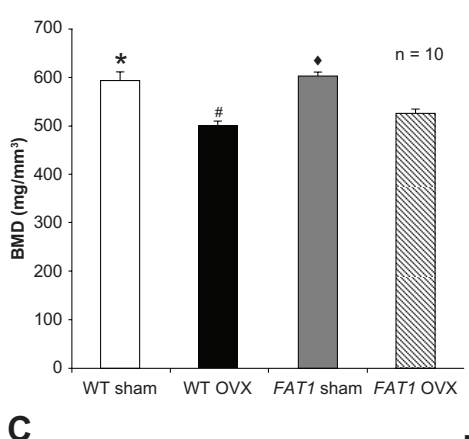

C

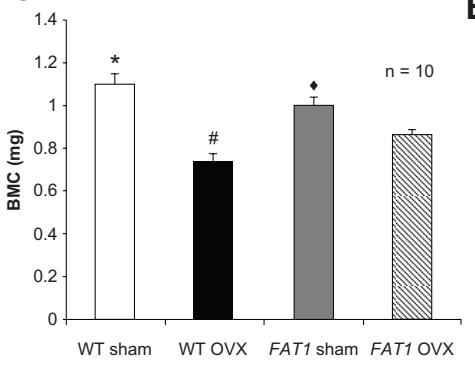

D



E 7.2

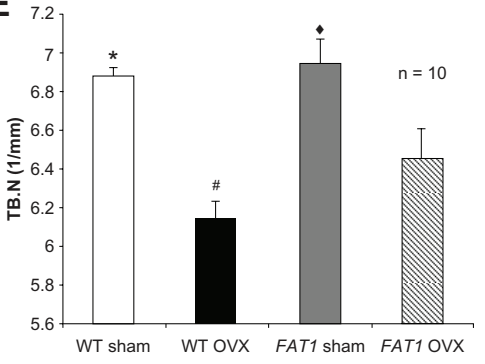

Figure 3 (A-E) Bone parameter assessment by micro-computed tomography scan. (A) Three-dimensional reconstructions of the distal femoral metaphysis of FATI and wildtype (WT) mice by micro-CT scan. The FATI ovariectomized (OVX) group exhibited more bone mass than the WT OVX group. (B-D) Quantitation of bone parameters of distal femur bone. The values of bone mineral density (BMD, B), bone mineral content (BMC, C), bone volume/total volume (BV/TV, D), and trabecular number (Tb.N) (E) in the OVX group were decreased compared with those in the sham group. The bone parameter (BMD, BMC, BV/TV) values of the FATI OVX group were significantly higher than the WT OVX group, but the Tb.N in the FATI OVX group only mildly increased than WT OVX group.



For better understanding of the effect of endogenous n-3 PUFAs, TG mice with FATl gene overexpression were developed, which was able to show a much lower n-6:n-3 PUFA ratio of $\sim 1: 1$ compared to WT mice with ratio of $20-30: 1 .^{23}$ Therefore, we used FATl transgenic mice in our study to investigate bone marrow adipogenesis under osteoporosis situations for the first time, taking advantage of its steady endogenous n-3 PUFA ratio.

Since adipogenesis and osteoblastogenesis from BMSCs are regulated by key transcription factors - PPAR $\gamma$ and RUNX2, respectively - expression of same could determine the establishment of adipocytes or osteoblasts. In this study, we observed a significantly lower expression of PPAR $\gamma$ but higher RUNX2 expression in bone marrow of TG mice when compared to WT mice, which confirmed the inhibitory effect of n-3 PUFAs on bone marrow adipogenesis theoretically.

With regard to the effect of fat on bone, peripheral adipose tissue and bone marrow should be taken into consideration. It has been suggested peripheral adipose tissue influences bone through stresses caused by mechanical loading. ${ }^{35}$ Recent in vitro and in vivo studies have drawn a conclusion that lowering dietary the n-6:n-3 PUFA ratio is associated with lower intra-abdominal fat mass and lower area of adipocytes from retroperitoneal fat,$^{36}$ which suggests that n-3 PUFA additions can inhibit the formation of peripheral adipocytes hypertrophy and hyperplasia. However, the effect of endogenous n-3 PUFAs on bone marrow adiposity and their possible mechanism needs further investigation due to underlying endocrine functions in bone, since adipocytes in bone marrow are an important source of adipokines that could have a direct lipotoxic effect on BMSCs and osteoblasts, which leads to bone loss. ${ }^{13}$ Our results revealed that endogenous n-3 PUFAs not only suppress the whole body weight of mice but also inhibit adiposity accumulation in bone marrow, preventing bone loss induced by ovariectomy in mice, indicating the potential effects of endogenous n-3 PUFAs on BMSCs, the unique precursors of bone marrow adipocytes. Future studies should look into the differentiation-modulation mechanism of BMSCs by endogenous n-3 PUFAs.

It has been suggested the current antiosteoporosis drugs, such as strontium ranelate and risedronate, which have been widely used in the prevention and treatment of osteoporosis in postmenopausal women, ${ }^{37,38}$ could inhibit bone loss partially by retarding bone marrow adipogenesis, with alteration of the RUNX2/PPAR $\gamma$ balance during BMSC differentiation. This study on FATI transgenic mice provides compelling evidence that bone marrow adipose is the critical therapeutic target of osteoporosis, and endogenous n-3 PUFAs are pro- 
A

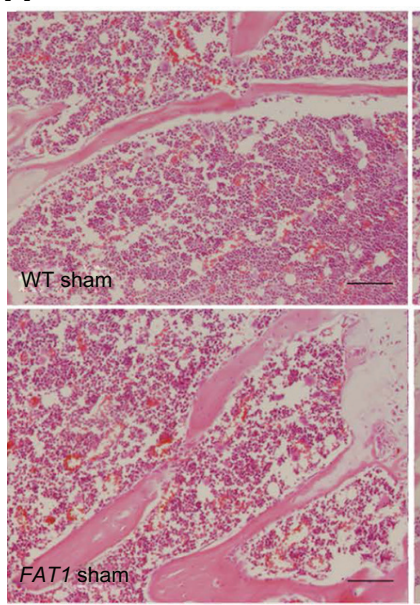

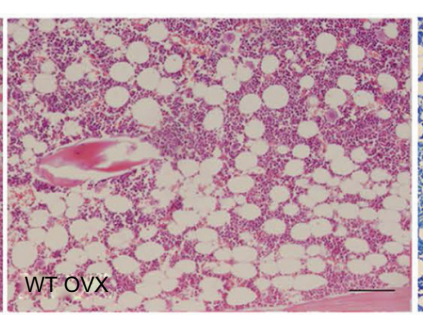

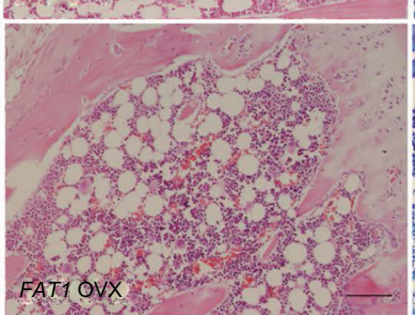

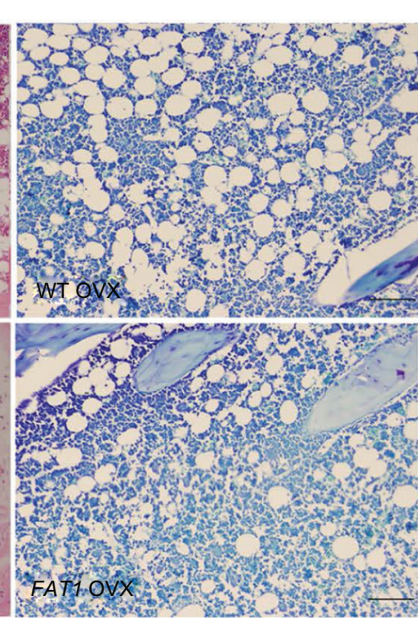

\section{B}

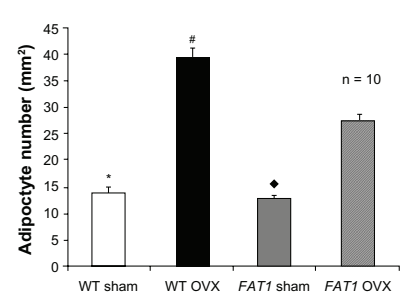

C

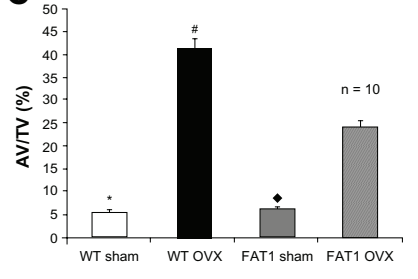

Figure 4 (A-C) Histological analysis of bone marrow adiposity. (A) Hematoxylin and eosin and toluidine blue staining for distal femur bone marrow. Images were taken at 200× magnification. There were fewer adipocytes in the FATI OVX group than the wild-type (WT) ovariectomized (OVX) group. (B) Adipocyte number (AD", per mm²) and $(\mathbf{C})$ adipocyte volume/tissue volume (AV/TV) were significantly reduced in the FATI OVX group. Three months after ovariectomy, the OVX group showed more bone marrow adiposity than the sham group $(P<0.05)$.

Notes: ${ }^{\#} P<0.05$ versus FATI OVX; ${ }^{*} P<0.05$ versus WT OVX; $\bullet P<0.05$ versus FATI OVX. Scale bars, $100 \mu \mathrm{m}$.

posed as a novel treatment in preventing postmenopausal osteoporosis.

Some hypotheses about the mechanisms by which n-3 PUFAs prevent bone mass loss associated with menopause have been proposed - enhancement of osteoblastogenesis and reduction of osteoclast activity - but these mechanisms are not yet fully understood. Combined with their effect of alleviation of proinflammatory cytokines such as interleukin 6 , interleukin 1 , and tumor necrosis factor- $\alpha$ secreted by bone marrow adipocytes ${ }^{39,40}$ and the effect of the marrow microenvironment modulating osteoclastogenic activity and increasing bone resorption, ${ }^{41-45}$ endogenous n-3 PUFAs,
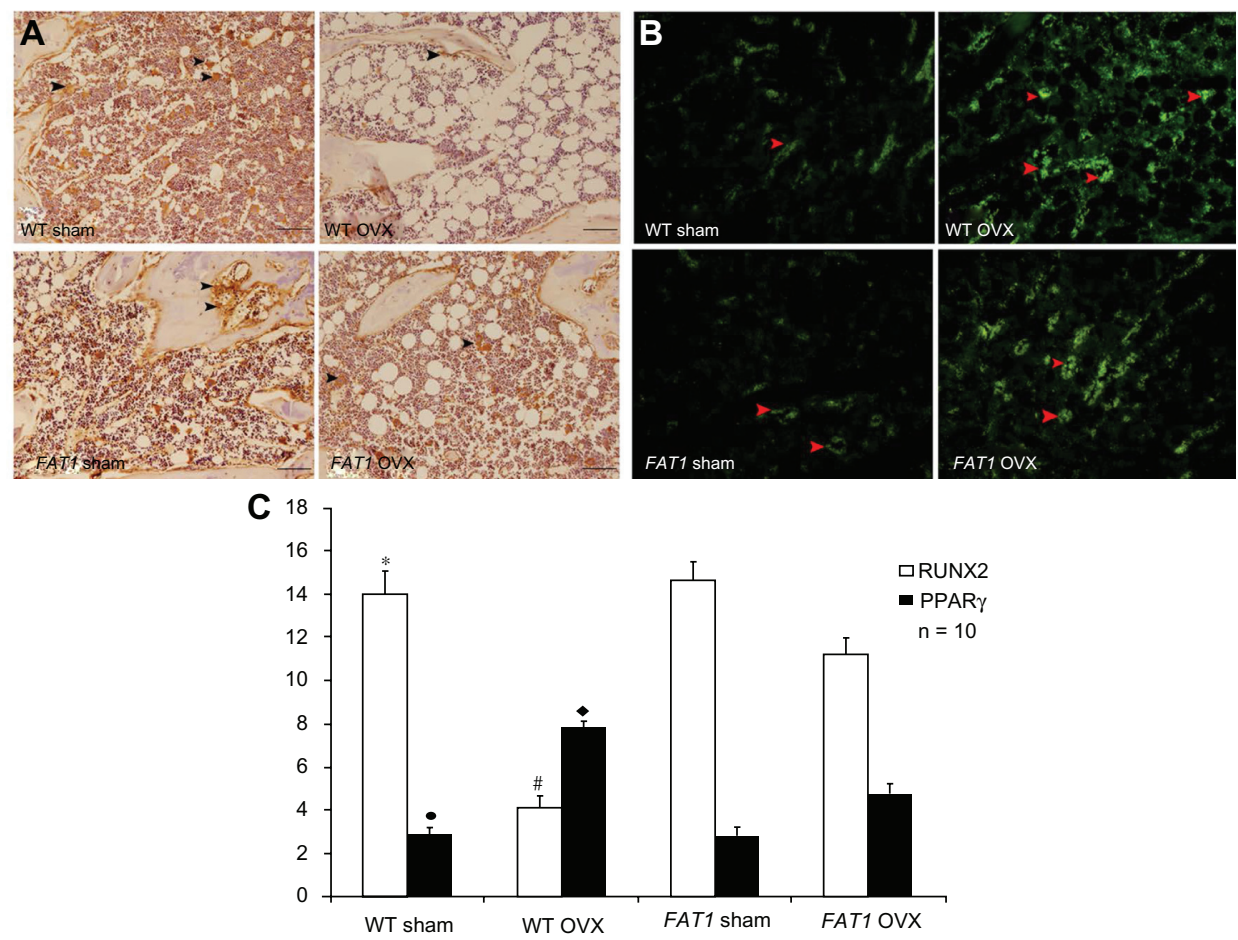

Figure 5 (A-C) Immunohistochemistry and counting of positively stained cells in bone marrow three months after ovariectomy. The black arrows indicate the runt-related transcription factor 2 (RUNX2) positively stained cells, and the red arrows indicate peroxisome proliferator-activated receptor gamma (PPAR $\gamma$ ) positively stained cells $(200 \times$ magnification, scale bar $=100 \mu \mathrm{m})(\mathbf{A}$ and $\mathbf{C})$. The proportion of RUNX2-positive cells was significantly increased in the FATI ovariectomized (OVX) group compared to wild-type (WT mice). (B and C) The proportion of PPAR $\gamma$-positive cells was significantly reduced in the FATI OVX group compared to WT mice. Notes: ${ }^{\sharp} P<0.05$ versus FATI OVX; ${ }^{*} P>0.05$ versus FATI sham; $\bullet P<0.05$ versus FATI OVX; $\bullet P>0.05$ versus $F A T I$ sham. Scale bars, $100 \mu$ m. 
which alleviated bone marrow adiposity in an osteoporosis animal model, may target bone marrow adipocytes by attenuating adipogenesis of BMSCs, subsequently increasing the osteoblastogenesis. However, the specific signaling pathway mechanism involved in this process should be further pursued.

In conclusion, in this study, we showed that the endogenous conversion of $n-6$ PUFA to $n-3$ PUFA and maintaining a lower ratio of n-6:n-3 PUFAs could attenuate adipogenesis within the bone marrow, which is associated with decreasing the level of PPAR $\gamma$ and increasing the level of RUNX2 expression.

\section{Conclusion}

Our findings provide us with a more comprehensive understanding of the mechanism of action of n-3 PUFAs on promoting osteoblastogenesis and perhaps will lead to the development of a therapeutic approach for osteoporosis aimed at reversing adipocyte differentiation from BMSCs.

\section{Disclosure}

The authors report no conflicts of interest in this work.

\section{References}

1. [No authors listed]. Consensus development conference: diagnosis, prophylaxis, and treatment of osteoporosis. Am J Med. 1993;94:646-650.

2. Meunier P, Aaron J, Edouard C, Vignon G. Osteoporosis and the replacement of cell populations of the marrow by adipose tissue. A quantitative study of 84 iliac bone biopsies. Clin Orthop Relat Res. 1971;80:147-154.

3. Griffith JF, Yeung DK, Antonio GE, et al. Vertebral marrow fat content and diffusion and perfusion indexes in women with varying bone density: MR evaluation. Radiology. 2006;241:831-838.

4. Verma S, Rajaratnam JH, Denton J, Hoyland JA, Byers RJ. Adipocytic proportion of bone marrow is inversely related to bone formation in osteoporosis. J Clin Pathol. 2002;55:693-698.

5. Owen M. Marrow stromal stem cells. J Cell Sci Suppl. 1988;10:63-76.

6. Rodríguez JP, Montecinos L, Ríos S, Reyes P, Martínez J. Mesenchymal stem cells from osteoporotic patients produce a type I collagen-deficient extracellular matrix favoring adipogenic differentiation. $J$ Cell Biochem. 2000;79:557-565.

7. Song L, Tuan RS. Transdifferentiation potential of human mesenchymal stem cells derived from bone marrow. FASEB J. 2004;18:980-982.

8. Muruganandan S, Roman AA, Sinal CJ. Adipocyte differentiation of bone marrow-derived mesenchymal stem cells: cross talk with the osteoblastogenic program. Cell Mol Life Sci. 2009;66:236-253.

9. Beresford JN, Bennett JH, Devlin C, Leboy PS, Owen ME. Evidence for an inverse relationship between the differentiation of adipocytic and osteogenic cells in rat marrow stromal cell cultures. J Cell Sci. 1992;102:341-351.

10. Clabaut A, Delplace S, Chauveau C, Hardouin P, Broux O. Human osteoblasts derived from mesenchymal stem cells express adipogenic markers upon coculture with bone marrow adipocytes. Differentiation. 2010;80:40-45.

11. Maurin AC, Chavassieux PM, Frappart L, Delmas PD, Serre CM, Meunier PJ. Influence of mature adipocytes on osteoblast proliferation in human primary cocultures. Bone. 2000;26:485-489.
12. Maurin AC, Chavassieux PM, Vericel E, Meunier PJ. Role of polyunsaturated fatty acids in the inhibitory effect of human adipocytes on osteoblastic proliferation. Bone. 2002;31:260-266.

13. Elbaz A, Wu X, Rivas D, Gimble JM, Duque G. Inhibition of fatty acid biosynthesis prevents adipocyte lipotoxicity on human osteoblasts in vitro. J Cell Mol Med. 2010;14:982-991.

14. Sakaguchi K, Morita I, Murota S. Relationship between the ability to support differentiation of osteoclast-like cells and adipogenesis in murine stromal cells derived from bone marrow. Prostaglandins Leukot Essent Fatty Acids. 2000;62:319-327.

15. Zevenbergen H, de Bree A, Zeelenberg M, Laitinen K, van Duijn G, Flöter E. Foods with a high fat quality are essential for healthy diets. Ann Nutr Metab. 2009;54:15-24.

16. Watkins BA, Li Y, Lippman HE, Seifert MF. Omega-3 polyunsaturated fatty acids and skeletal health. Exp Biol Med. 2001;226:485-497.

17. Fernandes G, Lawrence R, Sun D. Protective role of n-3 lipids and soy protein in osteoporosis. Prostaglandins Leukot Essent Fatty Acids. 2003;68:361-372.

18. Watkins BA, Li Y, Lippman HE, Feng S. Modulatory effect of omega-3 polyunsaturated fatty acids on osteoblast function and bone metabolism. Prostaglandins Leukot Essent Fatty Acids. 2003;68: 387-398.

19. Sun D, Krishnan A, Zaman K, Lawrence R, Bhattacharya A, Fernandes G. Dietary n-3 fatty acids decrease osteoclastogenesis and loss of bone mass in ovariectomized mice. J Bone Miner Res. 2003;18:1206-1216.

20. Green KH, Wong SC, Weiler HA. The effect of dietary n-3 long-chain polyunsaturated fatty acids on femur mineral density and biomarkers of bone metabolism in healthy, diabetic and dietary-restricted growing rats. Prostaglandins Leukot Essent Fatty Acids. 2004;71: 121-130.

21. Watkins BA, Reinwald S, Li Y, Seifert MF. Protective actions of soy isoflavones and n-3 PUFAs on bone mass in ovariectomized rats. J Nutr Biochem. 2005;16:479-488.

22. Shen CL, Yeh JK, Rasty J, Li Y, Watkins BA. Protective effect of dietary long-chain n-3 polyunsaturated fatty acids on bone loss in gonad-intact middle-aged male rats. Br J Nutr. 2006;95:462-468.

23. Rahman MM, Bhattacharya A, Banu J, Kang JX, Fernandes G. Endogenous n-3 fatty acids protect ovariectomy induced bone loss by attenuating osteoclastogenesis. J Cell Mol Med. 2009;13:1833-1844.

24. Wood RJ. Vitamin D and adipogenesis: new molecular insights. Nutr Rev. 2008;66:40-46.

25. Moerman EJ, Teng K, Lipschitz DA, Lecka-Czernik B. Aging activates adipogenic and suppresses osteogenic programs in mesenchymal marrow stroma/stem cells: the role of PPAR-gamma2 transcription factor and TGF-beta/BMP signaling pathways. Aging Cell. 2004;3:379-389.

26. Yoshida CA, Furuichi T, Fujita T, et al. Core-binding factor beta interacts with Runx 2 and is required for skeletal development. Nat Genet. 2002;32:633-638.

27. Wei D, Li J, Shen M, et al. Cellular production of n-3 PUFAs and reduction of n-6-to-n-3 ratios in the pancreatic beta-cells and islets enhance insulin secretion and confer protection against cytokine-induced cell death. Diabetes. 2010;59:471-478.

28. Elbaz A, Rivas D, Duque G. Effect of estrogens on bone marrow adipogenesis and Sirt1 in aging C57BL/6J mice. Biogerontology. 2009;10: $747-755$.

29. Syed FA, Oursler MJ, Hefferanm TE, Peterson JM, Riggs BL, Khosla S. Effects of estrogen therapy on bone marrow adipocytes in postmenopausal osteoporotic women. Osteoporos Int. 2008;19:1323-1330.

30. Meunier P, Aaron J, Edouard C, Vignon A. Osteoporosis and the replacement of cell populations of the marrow by adipose tissue. Clin Orthop Rel Res. 1971;80:147-154.

31. Justesen J, Stenderup K, Ebbesen EN, Mosekilde L, Steiniche T, Kassem M. Adipocyte tissue volume in bone marrow is increased with aging and in patients with osteoporosis. Biogerontology. 2001;2: $165-171$.

32. Kesavalu L, Vasudevan B, Raghu B, et al. Omega-3 fatty acid effect on alveolar bone loss in rats. J Dent Res. 2006;85:648-652. 
33. Bhattacharya A, Rahman M, Sun D, Fernandes G. Effect of fish oil on bone mineral density in aging C57BL/6 female mice. J Nutr Biochem. 2007; 18:372-379.

34. Lau BY, Ward WE, Kang JX, Ma DW. Femur EPA and DHA are correlated with femur biomechanical strength in young fat-1 mice. J Nutr Biochem. 2009;20:453-461.

35. Taes YE, Lapauw B, Vanbillemont G, et al. Fat mass is negatively associated with cortical bone size in young healthy male siblings. J Clin Endocrinol Metab. 2009;94:2325-2331.

36. Massiera F, Saint-Marc P, Seydoux J, et al. Arachidonic acid and prostacyclin signaling promote adipose tissue development: a human health concern? J Lipid Res. 2003;44:271-279.

37. Fournier C, Perrier A, Thomas M, et al. Reduction by strontium of the bone marrow adiposity in mice and repression of the adipogenic commitment of multipotent C3H10T1/2 cells. Bone. 2012;50:499-509.

38. Jin J, Wang L, Wang XK, et al. Risedronate inhibits bone marrow mesenchymal stem cell adipogenesis and switches RANKL/OPG ratio to impair osteoclast differentiation. J Surg Res. 2013;180:e21-e29.

39. Fried SK, Bunkin DA, Greenberg AS. Omental and subcutaneous adipose tissues of obese subjects release interleukin-6: depot difference and regulation by glucocorticoid. J Clin Endocrinol Metab. 1998;83: $847-850$.
40. Timper K, Grisouard J, Sauter NS, et al. Glucose-dependent insulinotropic polypeptide induces cytokine expression, lipolysis and insulin resistance in human adipocytes. Am J Physiol Endocrinol Metab. 2013;304:E1-E13.

41. Jilka RL, Hangoc G, Girasole G, et al. Increased osteoclast development after estrogen loss: mediation by interleukin-6. Science. 1992;257: 88-91.

42. Manolagas SC. Role of cytokines in bone resorption. Bone. 1995; 17(2 Suppl):63S-67S.

43. Lee YM, Fujikado N, Manaka H, Yasuda H, Iwakura Y. IL-1 plays an important role in the bone metabolism under physiological conditions. Int Immunol. 2010;22:805-816.

44. Hashizume M, Mihara M. The roles of interleukin-6 in the pathogenesis of rheumatoid arthritis. Arthritis. 2011;2011:765624.

45. Maurin AC, Chavassieux PM, Frappart L, Delmas PD, Serre CM, Meunier PJ. Influence of mature adipocytes on osteoblast proliferation in human primary cocultures. Bone. 2000;26:485-489.
Drug Design, Development and Therapy

\section{Publish your work in this journal}

Drug Design, Development and Therapy is an international, peerreviewed open-access journal that spans the spectrum of drug design and development through to clinical applications. Clinical outcomes, patient safety, and programs for the development and effective, safe, and sustained use of medicines are a feature of the journal, which

\section{Dovepress}

has also been accepted for indexing on PubMed Central. The manuscript management system is completely online and includes a very quick and fair peer-review system, which is all easy to use. Visit $\mathrm{http}: / /$ www.dovepress.com/testimonials.php to read real quotes from published authors.

Submit your manuscript here: http://www.dovepress.com/drug-design-development-and-therapy-journal 